\title{
Whole brain vascular imaging in a mouse model of Alzheimer's disease with two-photon microscopy
}

Patrick Delafontaine-Martel

Joel Lefebvre

Pier-Luc Tardif

Bernard I. Lévy

Philippe Pouliot

Frédéric Lesage 


\title{
Whole brain vascular imaging in a mouse model of Alzheimer's disease with two-photon microscopy
}

\author{
Patrick Delafontaine-Martel, ${ }^{\mathrm{a}, \star}$ Joel Lefebvre, ${ }^{\mathrm{a}}$ Pier-Luc Tardif, ${ }^{\mathrm{a}}$ Bernard I. Lévy, ${ }^{\mathrm{b}}$ Philippe Pouliot, ${ }^{\mathrm{a}, \mathrm{c}}$ and \\ Frédéric Lesage $\mathrm{e}^{\mathrm{a}, \mathrm{c}}$ \\ ${ }^{a}$ Ecole Polytechnique Montréal, Department of Electrical Engineering, Quebec, Canada \\ ${ }^{b}$ Vessels and Blood Institute, Inserm U970 and Hôpital Lariboisière, Paris, France \\ 'Montreal Heart Institute, Research Centre, Montreal, Quebec, Canada
}

\begin{abstract}
Given known correlations between vascular health and cognitive impairment, the development of tools to image microvasculature in the whole brain could help investigate these correlations. We explore the feasibility of using an automated serial two-photon microscope to image fluorescent gelatin-filled whole rodent brains in three-dimensions (3-D) with the goal of carrying group studies. Vascular density (VD) was computed using automatic segmentation combined with coregistration techniques to build a group-level vascular metric in the whole brain. Focusing on the medial prefrontal cortex, cerebral cortex, the olfactory bulb, and the hippocampal formation, we compared the VD of three age groups (2-, 4.5-, and 8-months-old), for both wild type mice and a transgenic model (APP/PS1) with pathology resembling Alzheimer's disease (AD). We report a general loss of VD caused by the aging process with a small VD increase in the diseased animals in the somatomotor and somatosensory cortical regions and the olfactory bulb, partly supported by MRI perfusion data. This study supports previous observations that $A D$ transgenic mice show a higher VD in specific regions compared with WT mice during the early and late stages of the disease (4.5 to 8 months), extending results to whole brain mapping. ๑ 2018 Society of Photo-Optical Instrumentation Engineers (SPIE) [DOI: 10.1117/1.JBO.23.7.076501]
\end{abstract}

Keywords: whole brain histology; two-photon fluorescence microscopic imaging; Alzheimer's disease.

Paper 180214R received Apr. 17, 2018; accepted for publication Jun. 21, 2018; published online Jul. 11, 2018.

\section{Introduction}

Cognitive impairment was long believed to be primarily due to decreased synaptic density and neuron loss. Recent findings have challenged this idea, suggesting disturbances in white matter (WM) to be a key factor as well. ${ }^{1}$ Considerable evidence is accumulating supporting an association between WM lesions and specific cognitive dysfunctions and/or dementia severity. ${ }^{2-13} \mathrm{An}$ association has also been established between vascular disorders, such as hypertension, elevated cholesterol, atherosclerosis, and cognitive impairments, ${ }^{14-16}$ and also between cardiovascular risk factors, and WM lesions. ${ }^{13,17-23}$ However, exploring the pathogenesis of vascular changes and cognitive impairment beyond the above associations remains difficult and subject to interpretation. It is not yet clear if there are causal effects or if common processes are involved in the pathophysiology. ${ }^{24-26}$

A correlation between vascular health and Alzheimer's disease $(\mathrm{AD})$ risk has also been established, with a higher probability of developing $\mathrm{AD}$ following exposure to vascular factors such as hypertension or vascular dementia caused by strokes. ${ }^{14,27,28}$ Understanding how brain vasculature is modified with $\mathrm{AD}$ as a function of age could help underpin these associations. In recent work, Cifuentes et al. ${ }^{29}$ used a dual-pathology model to investigate the cross talk between hypertension and $\mathrm{AD}$ in the APP/PS1 mouse and showed that hypertensive APP/PS1 mice have a deficit in episodic-like memory tasks, which was associated with increased amyloid deposits and

*Address all correspondence to: Patrick Delafontaine-Martel, E-mail: patrick .delafontaine-martel@polymtl.ca decreased microvascular density in the cortex, the medial prefrontal cortex, and the hippocampus. Without hypertension, such differences between APP/PS1 and wild type (WT) controls were subtler, decreased, and sometimes reversed (e.g., in the cortex). Given their correlation with amyloid deposits, characterizing these subtle changes in microvasculature at different ages in the whole brain could help characterize the spatiotemporal evolution of the AD pathology. However, such an investigation requires capturing all capillaries in the brain and the development of means to compare them at different time points at the same spatial location. Our goal in this work is to develop a methodology to enable such studies.

Ex-vivo imaging remains the gold standard for pathological evaluations of the brain but has historically been hampered by achievable spatial sampling limited by the tissue-slicing and slide-mounting process. Furthermore, deformations introduced by cutting make spatial comparisons difficult. Recent developments combining depth-resolved imaging with block-face imaging have improved this situation and enabled the threedimension (3-D) reconstruction of whole organs. Ragan et al. ${ }^{30}$ demonstrated that coupling a two-photon microscope with a vibratome and a mechanical stage enabled full 3-D fluorescent imaging of the mouse brain. Optical coherence tomography (OCT) was also demonstrated to yield contrasted images of $\mathrm{WM}^{31-34}$ that could be correlated to diffusion MRI.

In this work, two-photon microscopy (TPM) was combined with a vibratome to perform whole brain vascular imaging at high resolution. APP/PS1 and WT mice of 2, 4.5, and 8 months

$1083-3668 / 2018 / \$ 25.00$ @ 2018 SPIE 
were perfused at time of euthanasia with a FITC-gelatin perfusate to fill brain vasculature, and imaged serially. Combining images into a mosaic, 3-D brain reconstructions were built and coregistered with each other using tools adapted from magnetic resonance (MR) image processing. A brain atlas mask was then used to identify specific regions of interest (ROI). ${ }^{29}$ Quantification of microvascular density in ROIs, i.e., the hippocampus, the cortex, the olfactory bulb and the medial prefrontal cortex, was performed and compared with controls as a function of age. We then extended our results by building a local estimate of vascular density (VD) from raw images and performing whole brain comparison using statistical parametric mapping (SPM) techniques.

\section{Materials and Methods}

The Animal Research Ethics Committee of the Montreal Heart Institute approved all procedures, in accordance with the Canadian Council on Animal Care recommendations.

\subsection{Mice Groups}

For vascular imaging, heterozygous APP/PS1 and their control littermates (C57BL/6J, here referred to as WT) mice were divided into three age groups (2-, 4.5-, and 8-months-old). Each group consisted of $N=5$ animals for a total of $N=30$. APP/PS1 mice start developing AD around 4.5 months with full pathology presenting at 8 months. Two other groups of $21 \mathrm{WT}$ and $25 \mathrm{AD}$ mice at 6 months were also used to gather perfusion MRI estimates in the same species.

\subsection{Brain Perfusion and Fixation}

Animals were briefly anesthetized with $2 \%$ isoflurane and received intracardiac perfusion perfusion through the apex of the heart with $30-\mathrm{mL}$ phosphate buffered saline (PBS, $0.3 \mathrm{~mL} / \mathrm{min}$ ) followed by $30 \mathrm{~mL}$ of $4 \%$ paraformaldehyde (PFA) in PBS also at a rate of $0.3 \mathrm{~mL} / \mathrm{min}$ and then perfused with an FITC-gelatin perfusate $(20 \mathrm{~mL}$ at $0.6 \mathrm{~mL} / \mathrm{min}, 2 \%$ porcine skin gelatin type A (G1890, Sigma), $1 \%$ fluoresceinlabeled-albumin (A9771, Sigma) with the body tilted to 30-deg head down in supine position. At the last step, the mouse head was submerged in ice water with the heart clamped to ensure gel solidification. After 15 min of cooling, the brain was carefully retrieved and kept in PFA for later imaging.

\subsection{Imaging System}

The imaging system used a femtosecond dual beam laser (Insight DS+, Spectra Physics) with tunable range from 680 to $1300 \mathrm{~nm}$. A motorized $X Y$-stage (Zaber T-Series LSR150B) allowed moving the sample for mosaic acquisitions, to cover the entire sample. The objective used was XLFluor $4 \times / 340$, NA 0.28, WD $29.5 \mathrm{~mm}$ (Olympus, Japan). Sample $Z$-positioning was controlled by a motorized labjack (MLJ050, Thorlabs). The scanning system consisted of galvo mirrors (GVS002, Thorlabs) coupled by two converging lenses to place both mirrors at the scanning distance of the scan objective to reduce beam "walking" on the imaging-objective back aperture. A $4 \times$ beam expander was installed behind the galvo mirrors to fill the imaging-objective aperture with the telescope. A variable attenuator consisting of a polarizing cube and half-wave plate controlled the incoming power to the microscope. To ensure a constant signal throughout the tissue, laser power was controlled during the acquisition to correct for attenuation. A custom-made vibratome was installed to cut sample blocks. Fluorescence signal detection was done following a dichroic long pass (Chroma, 695dcxxr) and a bandpass filter (ET525/ $50 \mathrm{M}-2 \mathrm{P}$, Chroma Technology) placed in front of the photomultiplier tube (Hamamatsu PMT). After the acquisition of a complete slice, a section of $200 \mu \mathrm{m}$ was removed to expose a new surface to the microscope. Figure 1 shows an overview of the apparatus.

Imaging of the sample was implemented by combining images from different physical positions set by the $X Y$ stages. The raw images were taken with a $20 \%$ overlap in the $X Y$ plane and a $25 \%$ overlap in the depth $Z$-axis to ensure continuity in the stitching reconstruction. The microscope then proceeded automatically through the tiles, followed by vibratome sectioning, repeating the procedure until the whole brain was imaged. An algorithm was implemented to detect the cut sample surface using the maximal fluorescence signal received from a complete A-scan (moving the objective along $Z$ ). Measuring the surface at multiple locations, a plane was fit to the data and was used for image stack acquisition. As the vibratome did not cut a perfectly straight plane parallel to the imaging focal plane, this scan allowed modeling the imaging plane tilt along the entire sample to ensure that all the sample was imaged uniformly.

\subsection{Characterization of the Imaging System}

To measure the axial resolution of the microscope, 4- $\mu \mathrm{m}$ diameter fluorescent beads (F36909 FocalCheckTM fluorescence microscope test slide \#1, ThermoFisher) were scanned. An imaging sequence with $z$-steps of $10 \mu \mathrm{m}$ was performed and analyzed. To compute the lateral resolution of the system, a target (R1L1S1N, Negative 1951 USAF Target, ThorLabs) was used. The edge spread function (ESF) from an edge of the target lines was used for lateral resolution estimation with a digital step of $0.1 \times 0.1 \mu \mathrm{m}^{2}$ to ensure an image limited by optical resolution. See Fig. 2 for more details.

\subsection{Image Reconstruction}

Reconstruction consisted in transformations on raw tile data, to form a full 3-D image. The raw data were labeled on a 3-D grid to perform a tile registration optimization using a phasecorrelation pairwise registration method inspired by Preibisch et al. $^{35}$ and developed by Lefebvre et al. ${ }^{31}$ Stitching of the $X Y$ planes was done using a Laplacian diffusion method with a blending width of 0.2 in units of overlap size fraction. ${ }^{31}$ After reconstruction, a maximum intensity profile (MIP) was applied on 250- $\mu$ m-thick slabs. In some instances, we discarded unclear slices caused by issues described later in this report.

\subsection{Coregistration Method and Vascular Template Construction}

As a first step, the imaged brains were digitally downsized to $25 \times 25 \times 25 \mu \mathrm{m}^{3}$ resolution. Advanced normalization tools (ANTs) were used for coregistration to a template from the Allen Brain Institute using rigid followed by nonlinear registration. ${ }^{36,37}$ As a second step, as a custom template typically outperforms using a generic template ${ }^{38}$ these coregistered data were used as input to an algorithm inspired from Refs. 39 and 40 to create a vascular template from our own data. By including the mirror images of the 30 brains, a grand total of 60 brains 


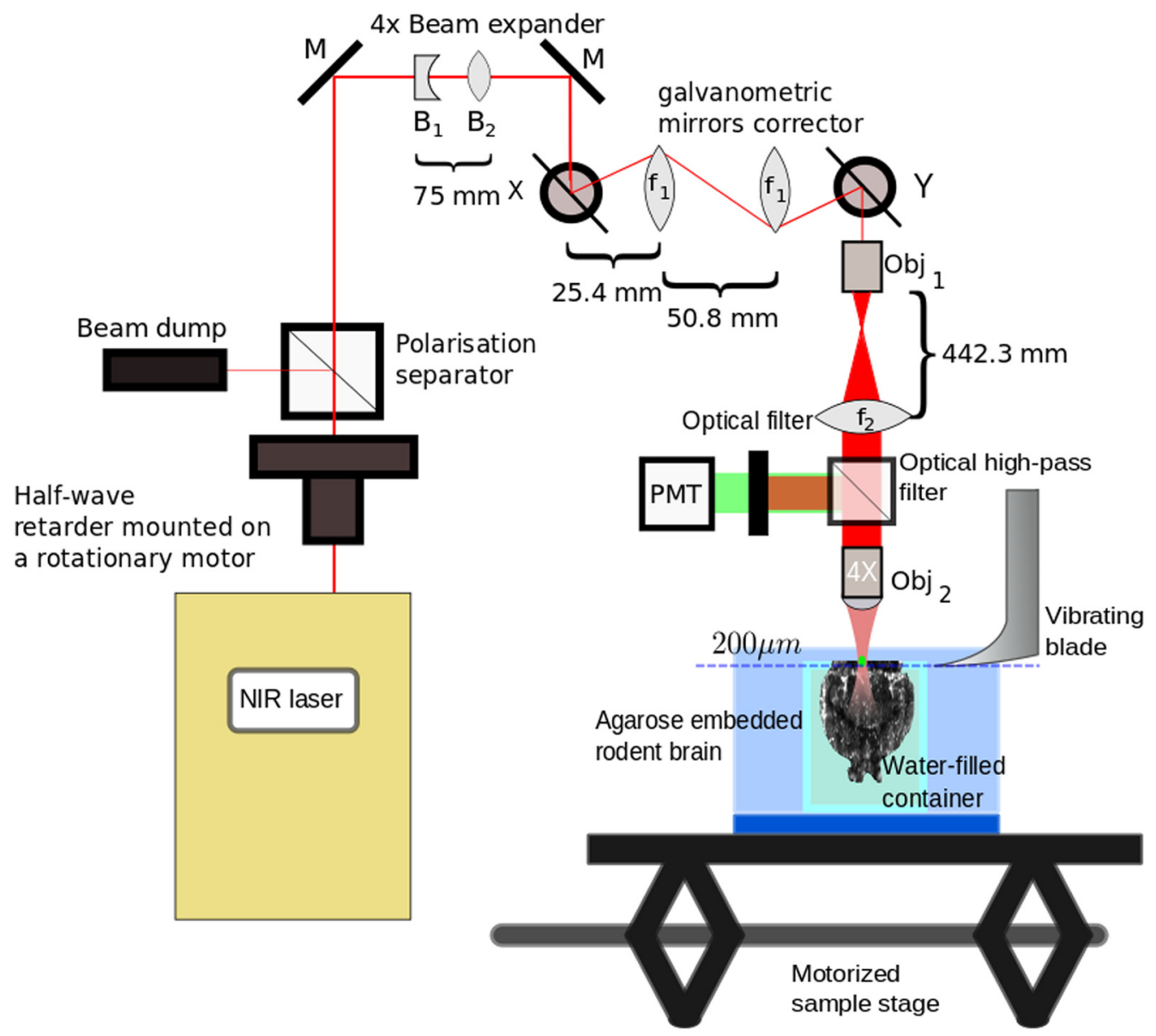

Fig. 1 Overview of the serial two-photon microscope. Legend: $B_{1}$ : $-25 \mathrm{~mm}$, LC1054; $B_{2}: 100.0 \mathrm{~mm}$, LA1207-B; $f_{1}: 25.4 \mathrm{~mm}$ AC254-030-B Lens (Thorlabs); $f_{2}: 400 \mathrm{~mm}$ focal AC508-400-B-ML Lens (Thorlabs); Obj ${ }_{1}$ : LSM04-BB, working distance $42.3 \mathrm{~mm}$; and $\mathrm{Obj}_{2}$ : XL Fluor $4 \times$ objective; M: mirrors.

were coregistered to form a vascular template, which was also coregistered to the Allen template. The ROIs defined from the Allen Brain Institute mouse template were then deformed onto each brain through the transformations defined by the deformation fields, and used for evaluations below.

\subsection{Vessel Extraction and Volume Computation}

Large vessels (15 $\mu \mathrm{m}$ in diameter or larger) were segmented using a variable thresholding based on local image properties by a local Otsu threshold on image patches 1501 pixels or $3002-\mu \mathrm{m}$ large, followed by a small median filter $(2 \times 2$ pixels or $4 \times 4 \mu \mathrm{m}$ ) to reduce segmentation noise. For smaller vessels, a Sato filter using a diameter of $2 \mu \mathrm{m}$ was applied. ${ }^{41}$ As the latter method can only distinguish eigenvalues in the data, it can underline the contour of larger vessels causing a "webbing" artifact (see Fig. 5). Combining both filters resulted in a multiscaled image with contrast for both small and large vessels in the imaged organ. After the segmentation process, a manual verification was performed to correct major deformations that could have been due to artifacts such as leakage of the vascular system causing diffusion zones in the sample. After this last step, and keeping only microvasculature ( $15 \mu \mathrm{m}$ in diameter or less), a distance transform was applied on the mask followed by skeletonization. To compute vascular density, a model of cylindrical vessels using the pixel value as radii and the resolution as length was computed based on the distance map evaluated on the skeleton.

\subsection{Whole Brain Local Vascular Density Comparison}

To investigate whether VD changes had a spatial structure, we estimated local VD in small-windowed regions of $200 \mu \mathrm{m}$ and built reduced-resolution VD images $\left(200 \times 200 \times 200 \mu \mathrm{m}^{3}\right)$. These images were then mapped to the vascular template to ensure a correspondence between each voxel. Voxel-wise analysis was performed with a two-way analysis of variance (ANOVA) with age and the genetic strain as factors, using the SPM12 toolbox. ${ }^{42}$ The ANOVA results showed no interaction and a statistically weak main effect of the genetic strain over the whole cortex, consistent with the analysis of Cifuentes et al. ${ }^{29}$ Therefore, the ROIs in Cifuentes et al., namely the hippocampal formation, the medial prefrontal cortex (mpfc), and cerebral cortex without the mpfc, were chosen to pursue the analysis, to which we added also the olfactory bulb based on the voxel-wise ANOVA.

\subsection{MRI Data for Comparison}

To assess whether VD changes had MRI correlates, we compared our vascular density TPM results with perfusion acquisitions. Twenty-one WT and 25 AD mice all aged 6 months (midtime point compared to the 4.5 and 8 months groups used for TPM) were imaged in an MRI scanner. Anatomical and perfusion MRI scans were performed on a 30-cm 7T horizontal MR 

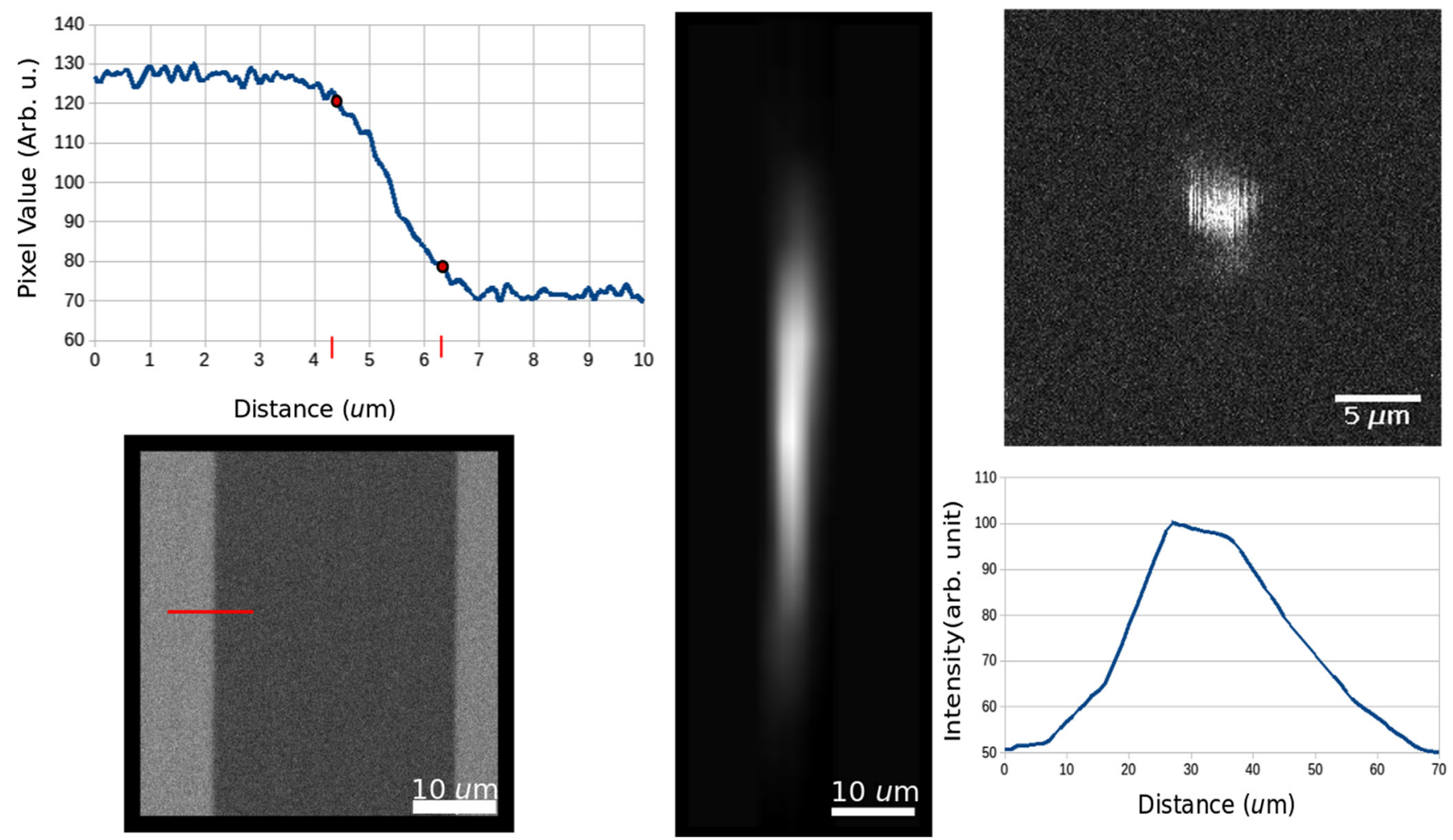

Fig. 2 ESF profile (top left) showing $10 \%$ and $90 \%$ points on the original image (bottom left). The distance separating these points is $2.1 \mu \mathrm{m}$. Side view of the PSF shows the elongated form given by the $4 \times$ objective (middle). Top view of an imaged bead (top right). The profile of the bead long axis yields an FWHM of $25 \mu \mathrm{m}$.

scanner (Agilent, Palo Alto, California) with mice in prone position, with a $12-\mathrm{cm}$ inner diameter gradient coil insert, gradient strength $600 \mathrm{mT} / \mathrm{m}$, and rise time $130 \mu \mathrm{s}$. A two-channel receive only surface coil positioned over the mouse brain was used in combination with a quadrature transmit/receive birdcage coil with an internal diameter of $69 \mathrm{~mm}$ (RAPID Biomedical, Germany). Anesthesia was maintained with $1.5 \%$ to $2.5 \%$ isoflurane in $30 \%$ oxygen in air and body temperature was maintained at $37.0^{\circ} \mathrm{C}$ using a warm air fan (SA Instruments, Stony Brook, New York). Respiration (target $=100$, allowed range before adjusting isoflurane $=80$ to $120 \mathrm{BPM}$ ) and heart rate were monitored, the latter with a pulse oximeter.

An anatomical image was acquired with a 3-D true free induction with steady-state precession (TFISP) sequence ${ }^{43}$ at $100-\mu \mathrm{m}$ isotropic resolution $(\mathrm{TR}=5.0 \mathrm{~ms} / \mathrm{TE}=2.5 \mathrm{~ms}, 16$ frequency shifts, 22-min scan time), used for coregistration. Then, a 3-D amplitude-modulated continuous arterial spin labeling scan (amCASL) was run, ${ }^{44}$ with TR $=3.0 \mathrm{~s}, 1.0$-s labeling duration, $60 \times 54 \times 48$ matrix, $300-\times 333-\times 333-\mu$ m resolution, and 23 mins scan time. The tagging plane was kept in a fixed position nominally perpendicular to the carotids.

\subsection{MRI Data Analysis}

Perfusion was calculated voxel by voxel using formula 1 in Ref. 44, with some parameters assumed constant: brain/blood partition coefficient $=0.9 \mathrm{~mL} / \mathrm{g}$, mouse arterial blood transit time $=0.08 \mathrm{~s}$, tagging efficacy $=0.67, T_{1 \mathrm{~b}}=2.3 \mathrm{~s}, T_{1}=1.53 \mathrm{~s}$, $T_{1 \mathrm{sat}}=0.57 \mathrm{~s}$, and $M_{a}^{z}(\mathrm{w})=0.48 \mathrm{~s}$. The anatomical scans were first manually coregistered rigidly using ITK-SNAP ${ }^{45}$ to a previously generated mouse template, then coregistered nonlinearly with ANTs. ${ }^{46}$ This provided the ANTs transformations to coregister the perfusion images. Coregistration results were inspected in detail, and registration parameters were stored as potential confounding variables. SPM ${ }^{42}$ voxel-by-voxel analyses were run on the perfusion images. The lack of any statistically strong effect suggested pursuing the analysis using the above four ROIs as for the ex vivo scanner study. The MarsBar ${ }^{47}$ toolbox in SPM was also used for the ROI statistics and to extract the average perfusion data.

\section{Results}

\subsection{Optical System Characterization}

As a first step, the two-photon microscopy system was evaluated using resolution targets described in Sec. 2.4. From fluorescent bead imaging and the ESF computation, the resolution of the optical system was measured to be $2.1 \mu \mathrm{m}$ in the lateral direction, and had a full width at half maximum (FWHM) of $25 \mu \mathrm{m}$ in $z$. This experimental resolution ensured that $50-\mu \mathrm{m} z$-motor steps during the acquisition procedure led to independent data from neighboring voxels.

\subsection{Raw Data Coregistration and Image Segmentation}

A vascular template was built from the data for each brain downsampled at an isotropic resolution $(25 \mu \mathrm{m})$. In this process, each brain was coregistered to this new template. The template, and hence each brain, were also coregistered to the Allen template. Figure 3 shows a typical coregistration for one brain.

Figure 4 shows the created template (B) aligned to the Allen template (A). Note that some sharp bright features are present in 


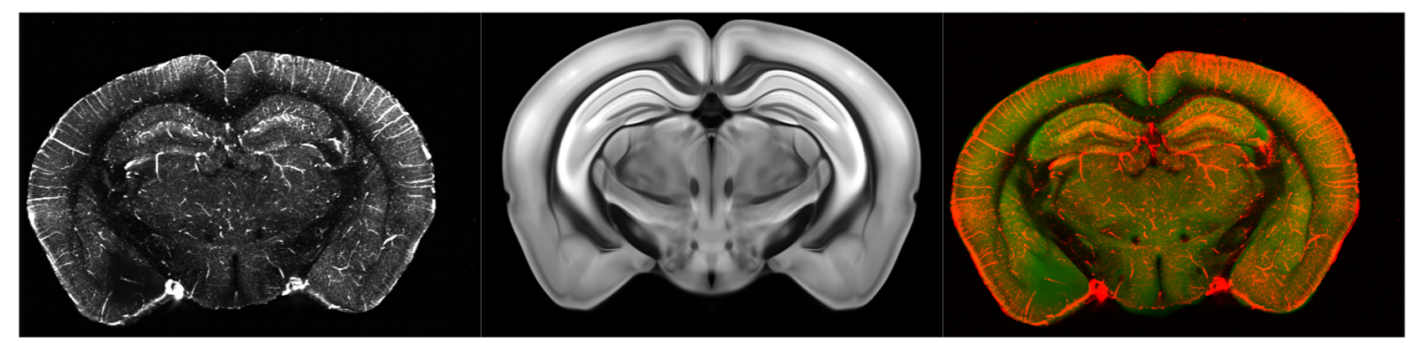

Data

(a)
Allen's template

(b)
Coregistration

(c)

Fig. 3 (a) Representative 250- $\mu$ m MIP from one mouse, reconstructed from the two-photon microscope data. (b) Brain template from the Allen's Brain Institute. (c) Overlay image representing the template (B in green) coregistered on the data (A in red) using the ANTs library.

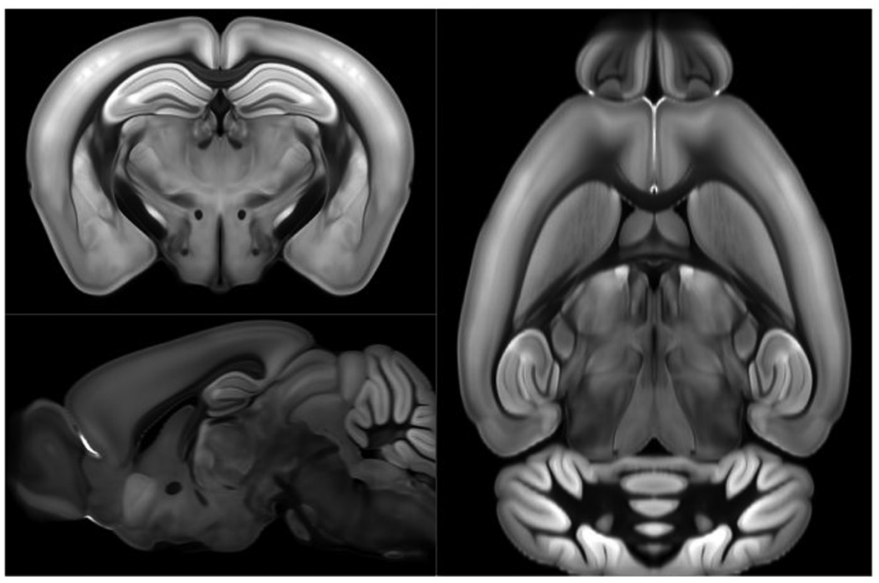

(a)

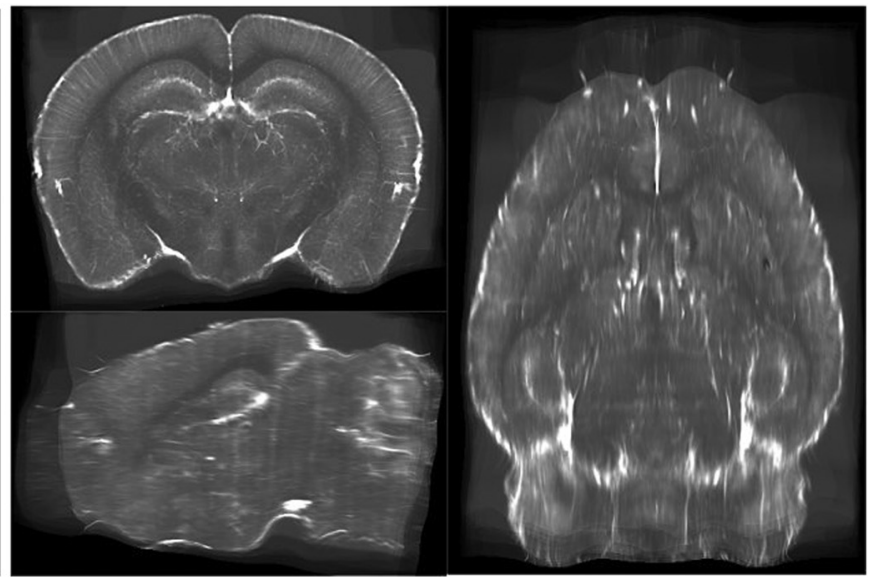

(b)

Fig. 4 Illustration of the Allen template with $25-\mu \mathrm{m}$ resolution (a) compared with the custom vascular template with the same resolution (b).

the vascular template. They were inspected to correspond to large blood vessels seen on many individual brains. We also observed that some regions of the olfactory bulb template have lower intensity. This corresponded to a few brains for which the bulb was incomplete. This may have arisen because all brains were cut along coronal sections with the olfactory bulb being the last surface to be exposed. Its mechanical stability in oxidized agarose was sometimes compromised, leading to data loss.

The ROIs derived from the Allen template atlas were mapped to the individual brains, using the transformation fields from template construction, and upsampled in the process to the original spatial resolution of the acquisition. These masks were then used for VD estimations derived from the segmentation process shown below.

The segmentation procedure described in Sec. 2.7 was applied on each brain using the full resolution. Figure 5 shows the results of this segmentation at intermediate steps, including the webbing effect in large vessels following Sato filtering and its correction through a mask combination step. The process yielded local estimates of vessel radius, which were used to compute VD with or without thresholds for vessel size.

\subsection{Voxel-Wise Statistical Parametric Mapping Analysis}

At $p<0.05$ uncorrected for false positives, results of the 2 ANOVA for VD showed a main effect of genetic strain covering the cortical area, the upper hippocampal complex, and some of the olfactory bulb. As discussed above, unfortunately, the olfactory bulb was truncated due to missing data for this structure and only the portion having complete overlap across all brains was kept for analysis. Based on this result, we selected the ROIs shown in Fig. 6 below.

\subsection{Regions of Interest Analysis}

Guided by previous observations using sparse sampling histology and the voxel-wise analysis above, we evaluated VD in four predefined ROIs: the hippocampal complex, the medial prefrontal cortex, the (truncated) olfactory bulb, and the cortex without the mpfc (Fig. 7).

Following the ROI mapping process and restricting to the microvasculature $(<15 \mu \mathrm{m})$ only, VD was computed and is shown in Fig. 8 for each ROI.

Two-way ANOVAs with age and genetic strain as factors showed a statistical significance for the mpfc and the olfactory bulb for the age factor, after Bonferroni correction for four regions, and removing two mice that were outliers. Posthoc tests showed that the VD decrease in 8-months-old mice compared with 2 months was significant for the above two ROIs. There, we also observed a tendency for VD decrease for both the olfactory bulb and the medial prefrontal cortex between 2 and 4.5 and for all ROIs between 4.5- and 8-months-old 


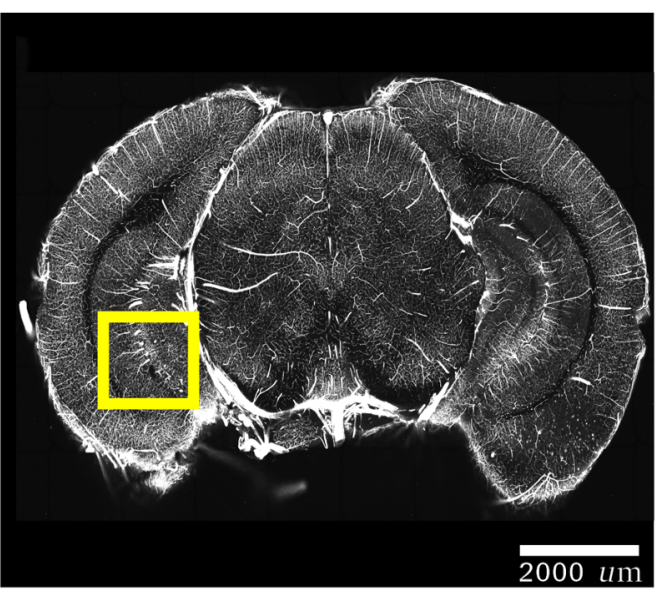

(a)

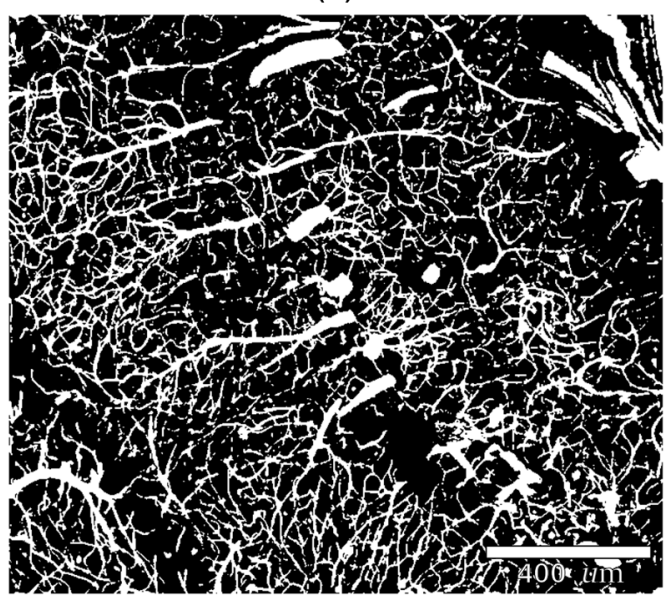

(c)

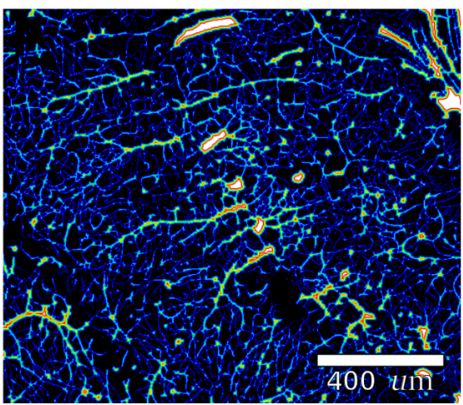

(e)

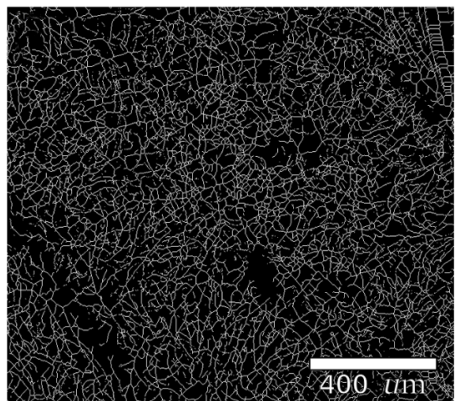

(f)

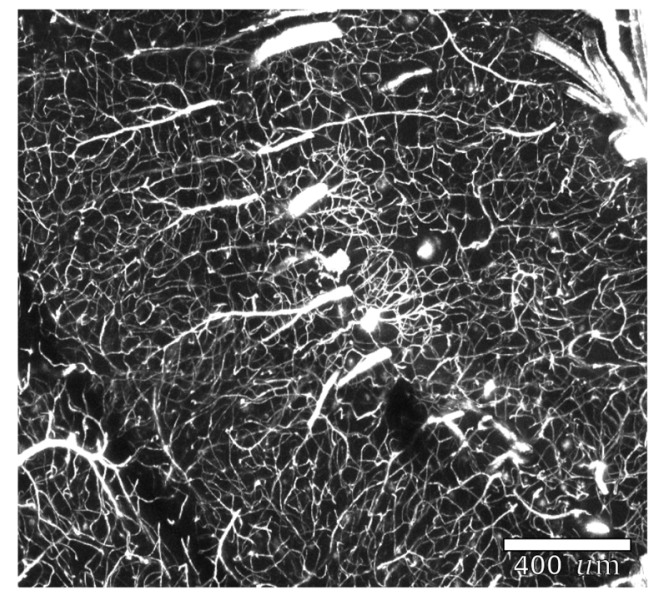

(b)

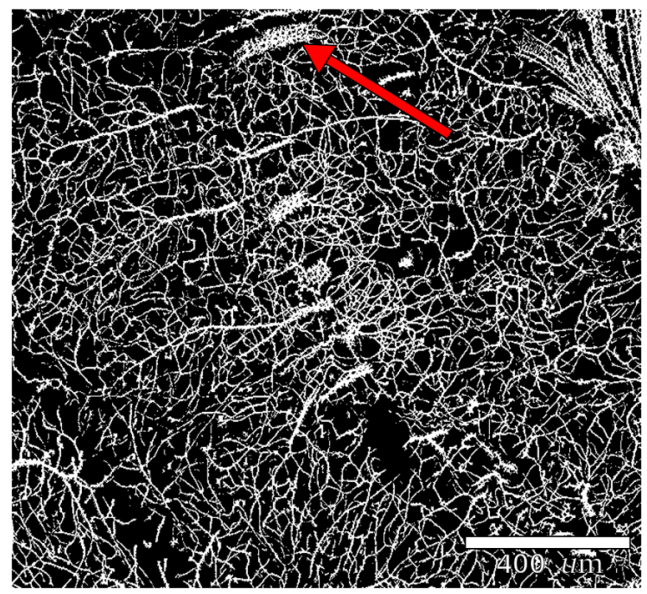

(d)

Fig. 5 Visualization of the segmentation algorithm (a) MIP from one brain. (b) Zoom on the inferior left hippocampal complex. (c) Local Otsu threshold applied on B. (d) Sato filter applied followed by an Otsu threshold. One can see the "webbing" vessels pointed by the red arrow of larger caused by the Sato filter. The local Otsu threshold combined with a median filter captures the larger blood vessels, whereas the Sato filter recovers smaller vessels with little salt-and-pepper noise. (e) A distance transformation is performed on the combination of the two masks and ( $f$ ) combined with the vessel center points derived from skeletonization by multiplication. $(\mathrm{g})$ As a result, we obtain an image in which pixels are the vessels radii.

for both APP/PS1 and WT mice. Furthermore, there was a tendency for higher VD in the APP/PS1 group when compared with the WT. The MRI perfusion data analyzed for comparison showed no statistical significance for the four ROIs. However, a nearly significant effect is present in the whole olfactory bulb ( $p=0.017$, APP/PS1 $>$ WT), correlating with a higher VD in the same region but that disappeared following Bonferroni.

\section{Discussion}

In this work, we developed a methodology that combined serial histology and 3-D reconstruction of microvasculature with whole-brain templates to investigate subtle changes in vasculature over most of the brain. Our work showed that it is possible to combine multiple serial acquisitions of microvasculature into a template that can then be used to map vascular density changes across the brain. 


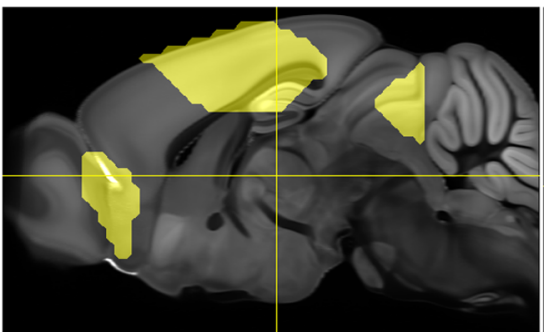

(a)

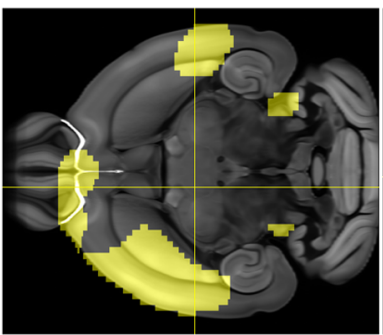

(b)

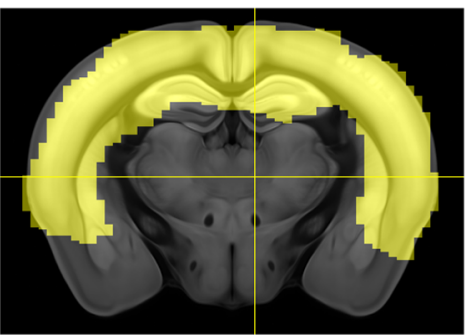

(c)

Fig. 6 (a) Sagittal view of the Allen template with Student's T-test positive for the contrast APP/PS1 > WT at $p<0.05$, uncorrected, (b) axial view, and (c) coronal view.

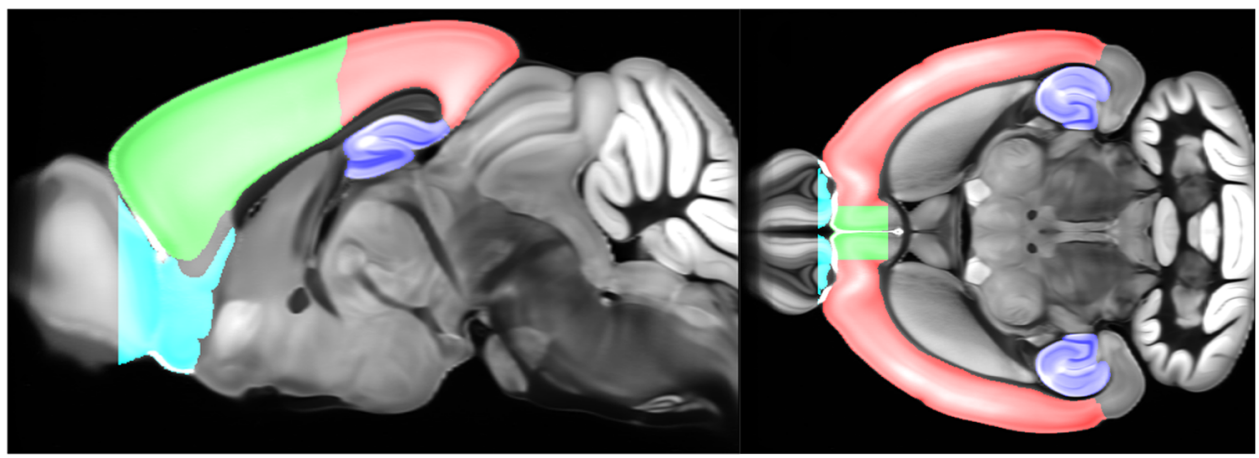

(a)

(b)

Fig. 7 (a) Sagittal view of the Allen template with the different ROIs (Hippocampal complex in blue, cortex in red, truncated olfactory bulb in cyan and medial prefrontal cortex in green). (b) Axial view of $A$.

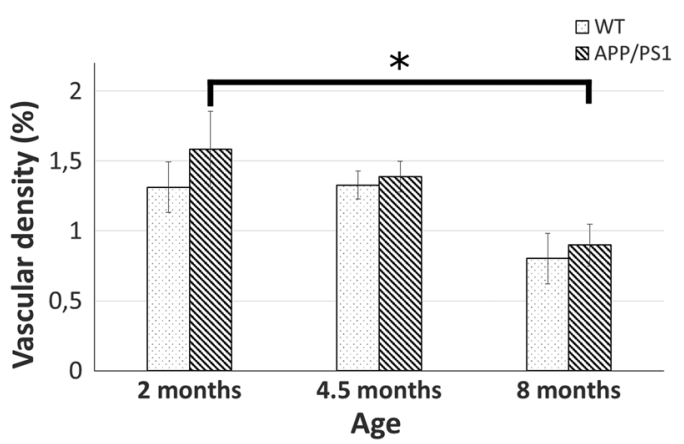

(a)

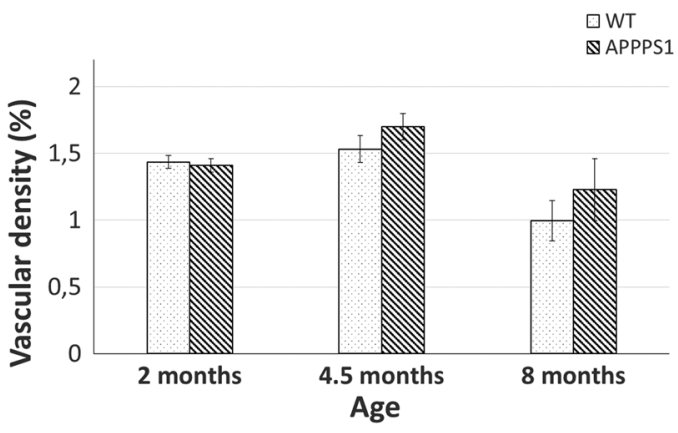

(c)

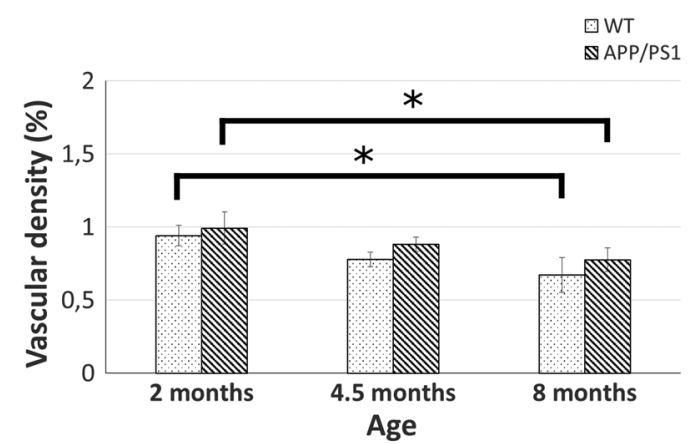

(b)

$\square$ WT $\mathbb{A} A P P / P S 1$

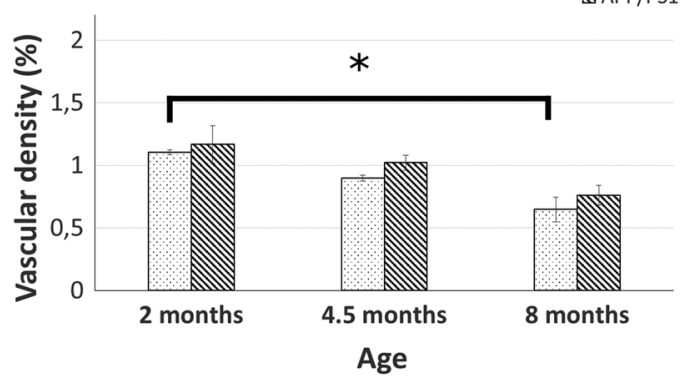

(d)

Fig. 8 Vascular density for each group and ROI. (a) Hippocampal ROI, (b) medial prefrontal cortex ROI. (c) Cortex without the mpfc. (d) Truncated olfactory bulb. One-tailed student T-tests were performed with $* p<0.05$ considered significant after Tukey-Kramer correction for the contrasts and Bonferroni correction for the four ROIs. Error bars represent one standard error on mean. 


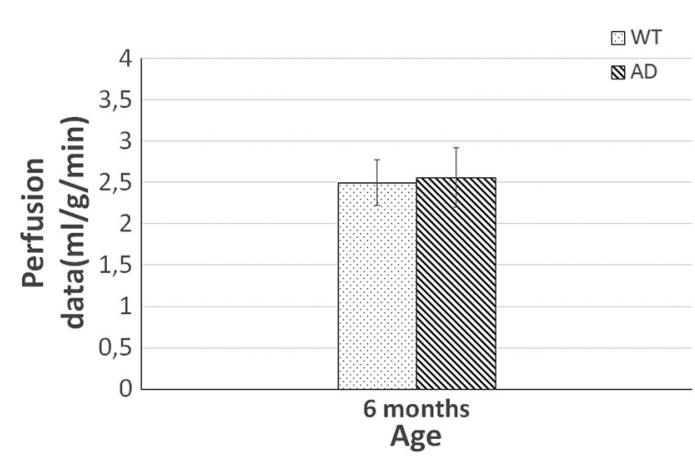

(a)

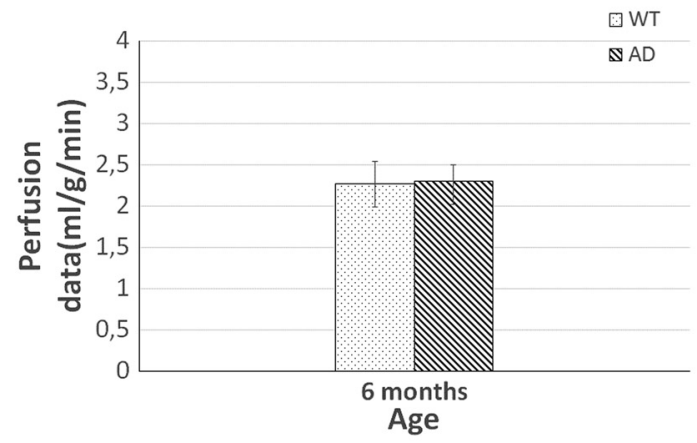

(c)

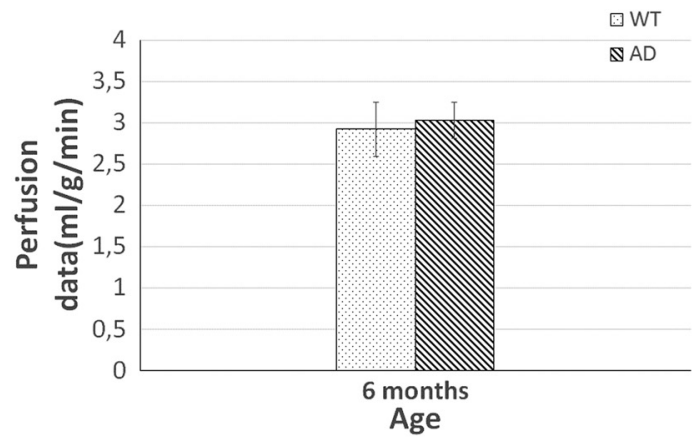

(b)

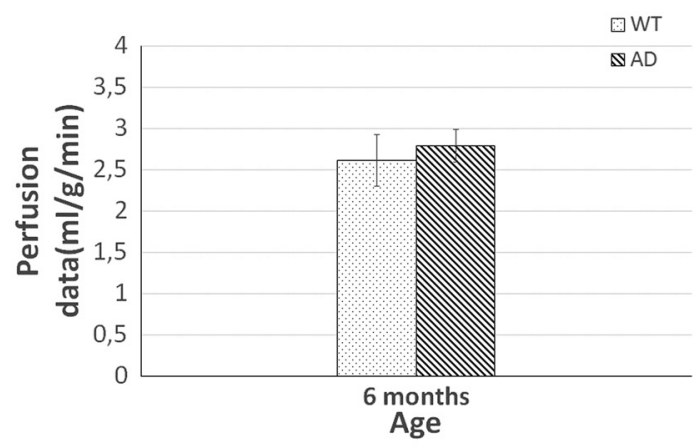

(d)

Fig. 9 Perfusion data from MRI. (a) Hippocampal complex, (b) medial prefrontal cortex, (c) Cortex without the medial prefrontal cortex, (d) Olfactory bulb. The ROls used are the same as used in the two-photon microscope. The pair differences are not statistically significant. However, when considering the whole olfactory bulb, the pair difference has an uncorrected $p$-value of $p=0.0017$, an effect that does not survive a Bonferroni correction.

A major challenge with our approach is consistent data acquisition. In some samples, the vibratome tissue cut was not optimal due to a misalignment of the blade. This led to defects in the form of zones where the imaging surface was uneven. This led to reduced resolution and sensitivity due to higher attenuation over the relatively thicker zones, impacting the VD estimates and leading to increased variance. In our data, these defects were mostly seen in the first samples from the 8-month-old groups, until the blade problem was fixed. Due to this, two of the 8-month-old brains could not be analyzed due to larger artifacts. Luckily, these brains were in different groups, distributing the loss equally for the 8-months-old brains.

As explained in Ref. 48, vascular density measurements are dependent on the segmentation process. In our case, using the Sato filter caused a thinning of the vessels contributing to an underestimation of vascular density. Moreover, as the axial resolution of the optical system is greater than the microvasculature diameter of interest $(<15 \mu \mathrm{m})$, superposition of vessels along the $z$-direction will happen at some points causing loss of data, which will also affect the overall VD measurements. However, capillaries are typically randomly oriented and by measuring a large number of slices, we obtain a high number of different configurations of vessels reflecting overall density. Indeed, due to the PSF, our estimates will underestimate VD, but comparison between groups should remain valid given that it will be constant across samples.

The local vascular analysis showed a tendency for a VD decrease in all ROIs except in the cerebral cortex, where an increase is observed as early as the start of the deposition of the $\mathrm{A} \beta$ plaques at 4.5 months. This tendency was significant when comparing 2- and 8-month samples for the medial prefrontal cortex and for one species in the olfactory bulb and hippocampus. The two-way ANOVAs performed on the ROIs pointed out that the age was nearly statistically significant, giving us insight that the aging process tends to diminish vascular density in mice. It should be noted that the data shown in Fig. 8 did not contain the complete olfactory bulb as sample extraction or the vibratome sequence caused some tissues to miss parts of the olfactory bulb. However, the clusters located at the frontal part of the brain contain most of the olfactory connections such as the Tenia tecta, the anterior commissure of the olfactory limb, and the anterior olfactory nucleus. It had been previously observed that a mouse model of Alzheimer's disease develops an impaired olfactory perceptual acuity, which could be explained by $\mathrm{A} \beta$ proteins deposition, ${ }^{48}$ which may be associated here with a vascular density decrease. Although consistent trends of higher VD were seen in the AD mice when compared with WT for all regions, no significant differences were seen. When comparing across species with an independent measure from MRI (Fig. 9), we also observed a trend of increased perfusion in the same areas, but still not significantly higher, in 6-month-old APP/PS1 mice compared with WT mice.

Voxel-wise analysis of the local VD maps suggests angiogenesis in cortical areas such as the somatomotor and somatosensory cortex of APP mice, which is also paired with an increased burden of $\mathrm{A} \beta$ deposition for older mice. ${ }^{49}$ Motor decline known as apraxia is a symptom for $\mathrm{AD}$ patients and transgenic mice. ${ }^{50}$ This observation is supported by the cerebral cortex VD in Fig. 8(c). 
Comparing our work with the results of Cifuentes et al., ${ }^{29}$ we observe the same tendencies of higher VD in AD mice except in the mpfc where Cifuentes et al. do not. Looking into their results shows a statistically significant change for the VD in the cortex, which could be a false positive due to smaller samples of images. The technique presented in this study contains a higher number of samples due to smaller slices imaged and a higher number of samples procuring numerous images thus giving a more powerful statistical analysis. Moreover, we included the olfactory bulb as an ROI, which was not considered in their previous study.

Even with the automatization implemented here, a disadvantage of the technique resides in the time required to prepare all the samples $(n=30)$. By upgrading the imaging system optical components, we could reduce the acquisition time significantly by a time factor of 4 . Such an upgrade will enable recording a higher number of samples and facilitate the study of subtle changes requiring higher statistical power. Moreover, with the presented technique, we could correlate the deposition of senile plaques using specific staining. Using a fluorophore with different emission spectra, local maps of senile plaques deposition could be coregistered on vascular changes, and one could observe if the vascular changes are locally correlated to plaques depositions. Another limitation of two-photon microscopy was the depth of penetration of light in the samples. Like many applications of such a system, the loss of resolution at higher depths can be a problem for morphological characterization. In our project, the maximal depth used was $250 \mu \mathrm{m}$ as we observed highly deteriorated images over this threshold. Using a spatial light modulator (SLM) could increase the depth by correcting the scattering of light. Another way to deal with scattering could be the CLARITY method to remove potential diffusor inside the biological sample. However, the acquisition method presented in this article is essential for further work where myelin sheets, cerebral white matter, or gray matter will be analyzed. Using the CLARITY technique in such a case would, therefore, remove or change significantly those lipid structures needed to understand the mechanisms of $\mathrm{AD}$.

\section{Conclusion}

We combined a two-photon microscope and a custom vibratome to perform block-face imaging of fluorescent gelatin-filled vessels. The imaging resolution was characterized to ensure that the system could measure blood vessel density. Following postprocessing segmentation to compute VD in APP/PS1 and wildtype brain samples, we investigated predefined ROIs (cerebral cortex, hippocampal formation, olfactory bulb, and medial prefrontal cortex) identified in previous studies to have vascular changes. Results point to a trend for an overall reduction of VD, which correlates with the mouse aging process.

For further investigations, we will include multimodal imaging such as coherent anti-Stokes Raman spectroscopy to obtain a contrast sensitive to white matter density and to correlate this contrast to a local vascular density map obtained by conjoined two-photon microscopy data, to gain more insight into the mechanisms underlying Alzheimer's disease.

\section{Disclosures}

The authors declare no competing financial interests.

\section{Acknowledgments}

This work was supported by CIHR and NSERC Discovery Grant program to F. Lesage, and by NSERC Discovery Grant, RGPIN-2014-06089 to P. Pouliot. A special thanks to Samuel Bélanger, Alexandre Castonguay and Maxime Abran for technical support. An earlier version is available as proceeding from the SPIE 2018 - BIOS conference. ${ }^{51}$

\section{References}

1. S. G. Kohama, D. L. Rosene, and L. S. Sherman, "Age-related changes in human and non-human primate white matter: from myelination disturbances to cognitive decline," AGE 34(5), 1093-1110 (2012).

2. M. Targosz-Gajniak et al., "Cerebral white matter lesions in patients with dementia-from MCI to severe Alzheimer's disease," J. Neurol. Sci. 283(1-2), 79-82 (2009).

3. N. D. Prins et al., "Cerebral white matter lesions and the risk of dementia," Arch. Neurol. 61(10), 1531-1534 (2004).

4. F. Fazekas et al., "The relation of cerebral magnetic resonance signal hyperintensities to Alzheimer's disease," J. Neurol. Sci. 142(1-2), 121-125 (1996)

5. R. Barber et al., "White matter lesions on magnetic resonance imaging in dementia with Lewy bodies, Alzheimer's disease, vascular dementia, and normal aging," J. Neurol. Neurosurg. Psychiatry 67(1), 66-72 (1999).

6. M. Yoshita et al., "Extent and distribution of white matter hyperintensities in normal aging, MCI, and AD," Neurology 67(12), 2192-2198 (2006).

7. J. C. de Groot et al., "Cerebral white matter lesions and cognitive function: the Rotterdam scan study," Ann. Neurol. 47(2), 145-151 (2000).

8. J. M. Ferro and S. Madureira, "Age-related white matter changes and cognitive impairment," J. Neurol. Sci. 203-204, 221-225 (2002).

9. D. W. Desmond, "Cognition and white matter lesions," Cerebrovasc. Dis. 13(Suppl. 2), 53-57 (2002).

10. M. M. Breteler et al., "Cognitive correlates of ventricular enlargement and cerebral white matter lesions on magnetic resonance imaging. The Rotterdam study," Stroke 25(6), 1109-1115 (1994).

11. B. C. Bowen et al., "MR signal abnormalities in memory disorder and dementia," Am. J. Roentgenol. 154(6), 1285-1292 (1990).

12. A. Kertesz, M. Polk, and T. Carr, "Cognition and white matter changes on magnetic resonance imaging in dementia," Arch. Neurol. 47(4), 387-391 (1990)

13. F. Fazekas et al., "MR signal abnormalities at $1.5 \mathrm{~T}$ in Alzheimer's dementia and normal aging," Am. J. Neuroradiol. 149, 351-356 (1987).

14. I. Skoog et al., "15-year longitudinal study of blood pressure and dementia," Lancet 347(9009), 1141-1145 (1996).

15. D. L. Sparks et al., "Increased incidence of neurofibrillary tangles (NFT) in non-demented individuals with hypertension," J. Neurol. Sci. 131(2), 162-169 (1995).

16. A. Hofman et al., "Atherosclerosis, apolipoprotein E, and prevalence of dementia and Alzheimer's disease in the Rotterdam study," Lancet 349(9046), 151-154 (1997).

17. F.-E. de Leeuw et al., "Aortic atherosclerosis at middle age predicts cerebral white matter lesions in the elderly," Stroke 31(2), 425-429 (2000).

18. M. Bots et al., "Cerebral white matter lesions and atherosclerosis in the Rotterdam study," Lancet 341(8855), 1232-1237 (1993).

19. A. E. George et al., "Leukoencephalopathy in normal and pathologic aging: 1. CT of brain lucencies," Am. J. Neuroradiol. 7(4), 561-566 (1986).

20. J. Van Swieten et al., "Hypodensity of the cerebral white matter in patients with transient ischemic attack or minor stroke: influence on the rate of subsequent stroke," Ann. Neurol. 32(2), 177-183 (1992).

21. W. D. Taylor et al., "Serial MR imaging of volumes of hyperintense white matter lesions in elderly patients: correlation with vascular risk factors," Am. J. Roentgenol. 181(2), 571-576 (2003).

22. G. Waldemar et al., "White matter magnetic resonance hyperintensities in dementia of the Alzheimer type: morphological and regional cerebral blood flow correlates," J. Neurol. Neurosurg. Psychiatry 57(12), 1458-1465 (1994) 
23. K. R. Bijanki et al., "Characterizing white matter health and organization in atherosclerotic vascular disease: a diffusion tensor imaging study," Psychiatry Res. Neuroimaging 214(3), 389-394 (2013).

24. C. Lecrux and E. Hamel, "The neurovascular unit in brain function and disease," Acta Physiol. 203(1), 47-59 (2011).

25. I. Segura et al., "The neurovascular link in health and disease: an update," Trends Mol. Med. 15(10), 439-451 (2009).

26. J. C. de la Torre, "Cerebrovascular and cardiovascular pathology in Alzheimer's disease," Int. Rev. Neurobiol. 84, 35-48 (2009).

27. M. Kivipelto et al., "Midlife vascular risk factors and Alzheimer's disease in later life: longitudinal, population based study," $\mathrm{Br}$. Med. J. 322(7300), 1447-1451 (2001).

28. H. Girouard, "Neurovascular coupling in the normal brain and in hypertension, stroke, and Alzheimer disease," J. Appl. Physiol. 100(1), 328-335 (2006).

29. D. Cifuentes et al., "Hypertension accelerates the progression of Alzheimer-like pathology in a mouse model of the disease," Hypertension 65(1), 218-224 (2015).

30. T. Ragan et al., "Serial two-photon tomography for automated ex vivo mouse brain imaging," Nat. Methods 9(3), 255-258 (2012).

31. J. Lefebvre et al., "Whole mouse brain imaging using optical coherence tomography: reconstruction, normalization, segmentation, and comparison with diffusion MRI," Neurophotonics 4(4), 041501 (2017).

32. A. Castonguay et al., "Serial optical coherence scanning reveals an association between cardiac function and the heart architecture in the aging rodent heart," Biomed. Opt. Express 8(11), 5027-5038 (2017).

33. H. Wang, J. Zhu, and T. Akkin, "Serial optical coherence scanner for large-scale brain imaging at microscopic resolution," Neurolmage $\mathbf{8 4}$, 1007-1017 (2014).

34. H. Wang et al., "Reconstructing micrometer-scale fiber pathways in the brain: multi-contrast optical coherence tomography based tractography," NeuroImage 58(4), 984-992 (2011).

35. S. Preibisch, S. Saalfeld, and P. Tomancak, "Globally optimal stitching of tiled 3D microscopic image acquisitions," Bioinformatics 25(11), 1463-1465 (2009).

36. A. Klein et al., "Evaluation of volume-based and surface-based brain image registration methods," NeuroImage 51(1), 214-220 (2010).

37. G. A. Johnson et al., "High-throughput morphologic phenotyping of the mouse brain with magnetic resonance histology," NeuroImage 37(1), 82-89 (2007).

38. L. Kuan et al., "Neuroinformatics of the Allen mouse brain connectivity atlas," Methods 73, 4-17 (2015).

39. A. L. Janke and J. F. P. Ullmann, "Robust methods to create ex vivo minimum deformation atlases for brain mapping," Methods 73, 18-26 (2015).
40. B. B. Avants et al., "The optimal template effect in hippocampus studies of diseased populations," Neurolmage 49(3), 2457-2466 (2010).

41. Y. Sato et al., "Three-dimensional multi-scale line filter for segmentation and visualization of curvilinear structures in medical images," Med. Image Anal. 2(2), 143-168 (1998).

42. C. V. Bowen, J. S. Gati, and R. S. Menon, "Robust prescan calibration for multiple spin-echo sequences: application to FSE and b-SSFP," Magn. Reson. Imaging 24(7), 857-867 (2006).

43. B. P. Chugh et al., "Robust method for $3 \mathrm{D}$ arterial spin labeling in mice," Magn. Reson. Med. 68(1), 98-106 (2012).

44. P. A. Yushkevich et al., "User-guided 3D active contour segmentation of anatomical structures: significantly improved efficiency and reliability," Neurolmage 31(3), 1116-1128 (2006).

45. B. B. Avants et al., "A reproducible evaluation of ANTs similarity metric performance in brain image registration," Neurolmage 54(3), 2033-2044 (2011).

46. K. J. Friston, "Statistical parametric mapping," in Neuroscience Databases, R. Kötter, Ed., pp. 237-250, Springer, Boston, Massachusetts (2003).

47. M. Brett et al., "Region of interest analysis using an SPM toolbox [Abstract]," in 8th Int. Conf. on Functional Mapping of the Human Brain, June 2-6, 2002, Sendai, Japan (2006).

48. D. W. Wesson et al., "Olfactory dysfunction correlates with amyloidburden in an Alzheimer's disease mouse model," J. Neurosci. 30(2), 505-514 (2010)

49. F. Trinchese et al., "Progressive age-related development of Alzheimerlike pathology in APP/PS1 mice," Ann. Neurol. 55(6), 801-814 (2004).

50. L. Giménez-Llort et al., "Modeling behavioral and neuronal symptoms of Alzheimer's disease in mice: a role for intraneuronal amyloid," Neurosci. Biobehav. Rev. 31(1), 125-147 (2007).

51. P. Delafontaine-Martel et al., "Large scale serial two-photon microscopy to investigate local vascular changes in whole rodent brain models of Alzheimer's disease," Proc. SPIE 10498, 1049820 (2018).

Patrick Delafontaine-Martel currently works at the Institute of Biomedical Engineering, Polytechnique Montréal. Their current project contains "serial histology" and "multiphotonic imaging" and he is currently studying for a PhD in biomedical engineering with the professor Frédéric Lesage's lab named Laboratoire d'Imagerie Optique et Moléculaire.

Biographies for the other authors are not available. 